\title{
Investigación como estrategia pedagógica para la convivencia escolar a través de competencias ciudadanas
}

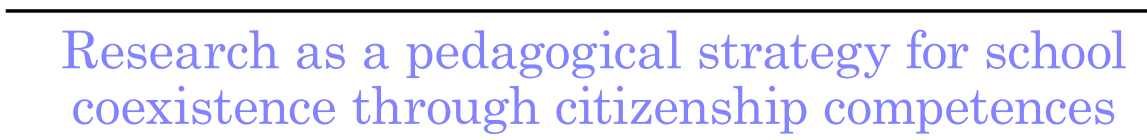

DOI: http://dx.doi.org/10.17981/cultedusoc.9.3.2018.33

Artículo de investigación. Fecha de recepción: 15/06/2018. Fecha de aceptación: 27/11/2018

\author{
Javier Camilo-Rojas; Alex Jiménez-Delgado; Blaneidis Jiménez-Jiménez; \\ Ibedis López-Delgado; Nibaldo Ospino-Ortiz; Mercedes Cabarcas-Massón; \\ Mirleidys Paba-Ruíz y Leticia Navarro-Ramírez ${ }^{1}$ \\ IED Antonio Bruges Carmona, sede Principal (Colombia) \\ jootta14@gmail.com
}

Para citar este artículo:

Camilo-Rojas, J., Jiménez-Delgado, A., Jiménez-Jiménez, B., López-Delgado, I., Ospino-Ortiz, N., Cabarcas-Massón, M., Paba-Ruíz, M. y Navarro-Ramírez, L. (2018). Investigación como estrategia pedagógica para la convivencia escolar a través de competencias ciudadanas. Cultura. Educación y Sociedad 9(3), 291-302. DOI: http://dx.doi.org/10.17981/cultedusoc.9.3.2018.33

\section{Resumen}

La educación en Colombia se ha orientado a la construcción de espacios democráticos con la finalidad de fortalecer la convivencia escolar, creando escenarios de participación para fomentar una educación en competencias ciudadanas. El estudio tuvo como propósito implementar la investigación como estrategia pedagógica para la convivencia escolar a través de competencias ciudadanas. Se guio bajo el enfoque cualitativo, con un diseño de Investigación Acción Participación (IAP), empleando técnicas de recolección como observación participante y diario de campo, se tomó una muestra representativa de sesenta (60) estudiantes de sexto grado con edades comprendidas entre diez (10) y trece (13) años de la institución educativa Antonio Bruges Carmona del municipio de Santa Ana - Magdalena. Los resultados arrojaron la implementación de espacios de participación mediante plataformas virtuales como redes sociales, concluyendo que la educación es clave para la transformación social hacia una cultura transparente donde la implementación de la investigación como estrategia pedagógica apoyadas en las TIC permite el fortalecimiento de la convivencia escolar y a su vez forma estudiantes con competencias ciudadanas.

Palabras clave: investigación como estrategia pedagógica, convivencia escolar, competencias ciudadanas.

\section{Abstract}

Education in Colombia has been oriented towards the construction of democratic spaces with the aim of strengthening school coexistence, creating participation scenarios to promote education in citizen skills. The purpose of the study was to implement research as a pedagogical strategy for school coexistence through citizenship competencies. It was guided under the qualitative approach, with a Participation Action Research (IAP) design, using collection techniques as participant observation and field diary, a representative sample of sixty (60) sixth grade students aged between ten (10) and thirteen (13) years of the educational institution Antonio Bruges Carmona of the municipality of Santa Ana - Magdalena. The results showed the implementation of participation spaces through virtual platforms such as social networks, concluding that education is key for social transformation towards a transparent culture where the implementation of research as a pedagogical strategy supported by TIC allows the strengthening of school coexistence and in turn trains students with citizenship competencies.

Keywords: research as a pedagogical strategy, school coexistence and citizenship skills.

1 Docentes de la IED Antonio Bruges Carmona, sede Principal, pertenecientes al grupo de investigación Filogenios hacia la politeia.

- The author; licensee Universidad de la Costa - CUC

Cultura, Educación y Sociedad vol. 9 no. 3, pp. 291-302. Diciembre, 2018

Barranquilla. ISSN 2389-7724 Online 


\section{Introducción}

La investigación es una forma precisa de encontrar respuestas que conduzcan a la reflexión sobre diversos temas, algunos específicos y otros amplios como la política. En respuesta a lo anterior surge la propuesta de integración curricular de la investigación como estrategia pedagógica, este estudio busco implementarla para fortalecer la convivencia escolar, generando espacios de participación en la escuela que fomenten la formación en competencias ciudadanas.

La Ley 115 (1994), conocida desde su expedición, como la Ley General de Educación, y el Decreto 1860 (1994), abrieron el sendero a las instituciones educativas colombianas para el aprovechamiento de una oportunidad valiosa orientada a la construcción de espacios democráticos, la formación de los estudiantes para la vida política y el ejercicio del arte de gobernar. Sin embargo, muy a pesar de que, a partir de la normatividad antes mencionada, las instituciones educativas dirigieron procesos electorales con tintes democráticos para cumplir con la Ley, particularmente, el artículo 142, que hace referencia a la "Conformación del gobierno escolar" (Ley 115, 1994, art. 142), prontamente los escenarios y prácticas estudiantiles se vieron mancilladas con los viejos vicios políticos que empañan la democracia de la ciudadanía legalmente constituida.

Por su parte la convivencia escolar es entendida como la acción de vivir en compañía con otras personas en el contexto escolar de forma pacífica y armónica. Hace referencia al conjunto de relaciones interpersonales que ocurre entre las personas que conforman la comunidad educativa, dichas relaciones deben enfocarse en el logro de objetivos educativos y su desarrollo integral (Guías pedagógicas para la convivencia escolar, 2013).
Las consecuencias notorias de estas prácticas viciadas y, desde luego, erradas, se evidencian en un ambiente escolar resquebrajado por comportamientos anti democráticos que fortalecen cada vez más la deteriorada sociedad santanera que se sumerge en unas prácticas que ha validado a través del tiempo, concibiendo que es la única forma en que puede y debe desarrollarse la política, generando la convicción de que las cosas tal como se dan, están bien, y que es imposible pensar y hacer algo distinto. Tristemente hay que reconocer que de la manera como acontece el ejercicio político por parte del colectivo estudiantil, no se es más que un útil semillero de electores y elegibles en potencia (Ochoa, 2015), presta a nutrir esa sociedad lejana del ideal anhelado, en donde reinen las habilidades y Competencias Ciudadanas que hagan posible un cambio de mentalidad y actitud.

De continuar por el sendero equivocado, no resta sino esperar para seguir siendo espectadores de un pálido reflejo de los pilares, de aquello que alguna vez fue soñado como democracia y muy distante de contar con verdaderos políticos o ciudadanos, en el amplio sentido de la expresión. El cómo evitar que esta situación problema siga su curso, sin ningún tipo de contención, es la tarea hacia la que se dirige la actividad de pensamiento del colectivo directivo y docente de la IED Antonio Bruges Carmona, que cree firmemente que es necesario un cambio de mentalidad, un cambio que no sólo es necesario, sino urgente.

Por esta razón apunta sus energías a diario hacia la orientación constante que pretende cambiar la forma de concebir la política y las prácticas en el desarrollo de actividades electorales, incluyendo el ejercicio honesto de las funciones asignadas por un cuerpo electoral. Por lo tanto, a partir del diseño de estrategias pedagógicas que, siendo aplicadas, contribuyan a la transformación de las prácticas democráticas y políticas de los niños y jóvenes 
de la institución, que, a su vez, tenga una repercusión en la vida social más allá de las cuatro paredes del entorno escolar.

En ese sentido la escuela se convierte en la mejor institución para generar una formación integral basada en la paz y los derechos humanos, sin embargo, esta no se encuentra aislada del entorno social, al contrario, es el lugar donde convergen todos los agentes de la comunidad. En realidad, los fenómenos de violencia escolar se han producido siempre, quizás con la misma o mayor intensidad. No obstante, ahora se han visibilizado más porque afectan a más personas, y porque los medios de comunicación, los padres y madres de los alumnos y la sociedad en general, se han sensibilizado frente a las problemáticas relacionadas con la educación (Colombo, 2011).

Las instituciones educativas son el medio más adecuado para formar ciudadanos integrales, democráticos, responsables y críticos, que contribuyan a la construcción de una sociedad justa, equitativa e igualitaria, donde se propicien espacios libres de violencia, basados en la sana convivencia y respeto a las diferencias individuales, promoviendo valores y fomentando el cumplimiento de normas sociales que posibiliten la convivencia de los estudiantes no solo en las instituciones educativas, sino en todos los entornos donde se desenvuelvan, propiciando una sociedad libre, democrática, abierta, multicultural, plural y globalizada (Gallardo, 2009).

En concordancia expresan Hernández, Alvarado y Chumaceiro (2013: 77), "La diversidad que caracteriza a las sociedades postmodernas, impulsa una participación inclusiva, con la afirmación de derechos plenos para todos como punta de lanza para asumir; la tolerancia como deber, entender la fragmentación multicultural en igualdad de condiciones, y lo complejo de las relaciones humanas para facilitarlas, y más aún, porque desde el reconocimiento de la otredad se hace espacio para el propio reconocimiento."

Para la formación de ciudadanos democráticos, se requiere un conjunto de competencias tales como; participación, cooperación, responsabilidad, tolerancia, solidaridad, habilidades sociales y gestión de conflictos interpersonales, así los estudiantes mediante equipos de trabajo tendrán la capacidad de gestionar democráticamente la resolución de conflictos que alteran la convivencia en la comunidad educativa, adoptando estrategias que permitan la participación de las personas, de tal manera que adquieran un compromiso por dialogar, escuchar, expresar sentimientos y participar en sus propios conflictos, sin desencadenar comportamientos violentos, tanto en el contexto escolar como en el social (Guzmán \& Preciado, 2012).

La educación para la paz y la convivencia debe servir como elemento integrador transversal a las asignaturas. Desde esta perspectiva, se deben incorporar en el plan de aula de los maestros acciones que fomenten una cultura de paz y sana convivencia, fortaleciéndolas en actividades específicas y métodos generales de su especialidad, ampliando el sentido educativo de la misma. Por lo cual los temas transversales introducen nuevos contenidos en el currículo, pero sobre todo reformulan y reintegran los existentes desde un nuevo enfoque integrador e interdisciplinar (Gutiérrez \& Pérez, 2015).

Así también, cuando se definen en la Ley General de Educación, los Fines de la educación, se destaca para el interés de la presente investigación, el numeral 2 del artículo 5, que expresa la Ley 115, (1994), art. 5: "La formación en el respeto a la vida y a los demás derechos humanos, a la paz, a los principios democráticos, de convivencia, pluralismo, justicia, solidaridad y equidad, así como el ejercicio de la tolerancia y de la libertad". 
Encontrando sustento, por cuanto la finalidad de la educación se concibe, en uno de sus apartes, como la necesidad de formar para la convivencia, en medio del pluralismo y la diversidad que se manifiesta en forma de condición social, diferencias ideológicas o religiosas, diferencia racial; en todo caso, el deber apunta a lograr una sana convivencia en la instituciones escolares, entendiendo que tal deber no se debe quedar en una mera ilustración catedrática sobre qué es o cómo debe ser la democracia, sino que su mirada debe ir más lejos: formar teóricamente con pretensiones prácticas para la vida política, cuidando de no tropezar con el error de enseñar para replicar una cultura política que debe alistarse para ser erradicada.

\section{Convivencia escolar y competencias ciudadanas mediante la IEP}

Es digno señalar que el marco sobre el que descansa uno de los mayores propósitos del Estado colombiano a través del Ministerio de Educación Nacional (MEN), es la Declaración Universal de los Derechos Humanos, en la medida que plantea la formación en Competencias Ciudadanas, que según su apreciación "es formar en y para los Derechos Humanos. El Ministerio de Educación Nacional, define las Competencias Ciudadanas en el entendido de un conjunto de habilidades cognitivas, emocionales y comunicativas que, integradas con conocimientos y actitudes, permiten que las personas se relacionen pacíficamente con otras y participen constructiva y democráticamente en una sociedad (MEN, 2005, p. 155); así, brinda una luz muy valiosa para la comprensión de los ambientes escolares, el rol del docente y la necesidad de formar a los niños, niñas y jóvenes para la ciudadanía.

Esto implica que el docente tenga la lucidez para dirigir sus esfuerzos en la concreción de tal finalidad, teniendo en cuenta que los ambientes escolares no son siempre el que se desea, sino que está acompañado de múltiples factores que afectan la convivencia entre estudiantes de una misma aula escolar y a la vez entre éstos y estudiantes de otras aulas; entre esos: la influencia del contexto familiar y social que determinan, como otra escuela, diversos tipos de comportamientos negativos o positivos, en una clara imitación de lo observado, incluyendo comportamientos de tipo político. Valiosa es la apreciación del filósofo y político Antanas Mockus, al señalar lo que significa las Competencias Ciudadanas:

Es necesario recalcar que, la ciudadanía es un mínimo de humanidad compartida. Cuando decimos que alguien es ciudadano, pensamos en aquel que respeta unos mínimos, que genera una confianza básica. Ser ciudadano es respetar los derechos de los demás. El núcleo central para ser ciudadano es, entonces, pensar en el otro. Se basa en tener claro que siempre hay otro, tener presente no sólo al otro que está cerca y con quien sabemos que vamos a relacionarnos directamente, sino también considerar al otro más remoto, al ser humano aparentemente más lejano-al desconocido, por ejemplo- o a quien hará parte de las futuras generaciones. pero el ciudadano también se define por su relación con el Estado. Uno es ciudadano de un país específico. Cuando se habla de las consecuencias, un ciudadano no sólo mira las consecuencias para unos, sino para todos, (Mockus, 2004). Cita paráfrasis.

En consecuencia, entendiendo que tal formación debe ser orientada al interior de las instituciones educativas del país, se expide en el año 2013, la Ley 1620, reconocida como Ley de Convivencia Escolar, que asume en uno de sus considerandos que:

uno de los retos que tiene el país, está en la formación para el ejercicio activo de la ciudadanía y de los Derechos Humanos, a través de una política que promueva y for- 
talezca la convivencia escolar, precisando que cada experiencia que los estudiantes vivan en los establecimientos educativos, es definitiva para el desarrollo de su personalidad y marcará sus formas de desarrollar y construir su proyecto de vida (Ley 1620, 2013, p. 1).

Es un reto inmenso, porque entra a jugar parte importante la idea de transformación, asumiendo la tarea de formar para un cambio que se reflejará en la vida social que adelantan los estudiantes a diario. De tal manera, que todo cuanto pueda contribuir para lograr este propósito será bienvenido y así fortalecer las actividades que en el marco de una investigación se puedan realizar, asumiendo que la convivencia en un determinado lugar está supeditada a muchos factores, entre los que cabe mencionar, el factor político que crea diferencias, divisiones, agresiones físicas y verbales, por la manera como se decide, se piensa o se gobierna.

En esa línea de conceptualización, el Ministerio de Educación Nacional, lidera un proceso de reglamentación participativa de la Ley 1620 de 2013, para cumplir con los compromisos y las responsabilidades que se establecen en ella. El producto de este proceso es la Guía Pedagógica para la Convivencia Escolar, más conocida como la Guía 49; en ella se recalca una vez más la preocupación del Estado por formar "en el ejercicio de la ciudadanía activa, reflexiva, crítica y participativa, que conviva pacíficamente y en unidad, como parte de una nación próspera, democrática e incluyente" (MEN, 2013, p. 5). La Ministra de Educación (2013) de aquel entonces, María Fernanda Campo Saavedra, en la presentación de la Guía 49, enfatizaba en la necesidad de construir ciudadanía; en sus palabras expresa:

Cabe decir que, que en gran parte somos conscientes que es necesario educar para convivir y participar valorando la diferencia y la diversidad; que la escuela y las familias son los primeros espacios sociales donde se crean las condiciones para que las niñas, niños y adolescentes desarrollen capacidades que les permiten expresar, discutir y disentir pensamientos, ideas y emociones, con argumentos claros, reconociendo y respetando otras formas de pensar, sentir y actuar es allí donde la ciudadanía se pone en práctica todos los días y se desarrollan las competencias necesarias para la transformación social.

Esta visión es pertinente para los intereses de todo proyecto de investigación que se presente, como es el caso de éste, buscando la construcción de espacios donde se brinden las condiciones para vivir en un ambiente de paz y de armonía. Resulta oportuno decir que, labor no resulta una tarea fácil, se hace compleja en la medida que en un aula escolar confluyen niñas, niños y adolescentes que viene de diversos contextos socio culturales, poseen diferentes intereses, entre otros aspectos que se pueden señalar, que determina el comportamiento individual con incidencia en lo colectivo; de acuerdo a esta apreciación, la Guía 49, es clara en manifestar que; formar para el ejercicio de la ciudadanía se entiende por tanto como un desafío para la escuela y para la sociedad colombiana, pues para lograr un verdadero ejercicio de la ciudadanía se requiere realizar cambios de tipo socio cultural que conviertan la memorización de contenidos en procesos pedagógicos críticos, (MEN, 2013, p. 13).

En esa línea de conceptualización se hace cada vez más claro que es menester el fundamentar las acciones en un pensamiento crítico que conlleve a desarrollar habilidades que hagan posible la comprensión entre miembros de la escuela, que adelanten su proceso de empoderamiento comprensivo de los acuerdos institucionales y del aula, tal como orienta la Guía: No basta con memorizar lo que contiene 
el manual de convivencia o conocer los protocolos de atención para mejorar el clima escolar, es necesario que la comunidad educativa participe y se apropie de los acuerdos allí contenidos y que estos faciliten el desarrollo de competencias como la empatía, toma de perspectiva o pensamiento crítico, los cuales son esenciales para un ejercicio real de la ciudadanía (MEN, 2013, p. 13).

Así pues, el ideal del Ministerio de Educación Nacional y el de los maestros de Colombia, que crean y anhelen una sociedad mejor es: "convertir a la escuela en un espacio de vivencia cotidiana de la democracia, donde se reconoce la dignidad humana como un valor supremo, y se puede participar y convivir en un marco de valoración de las diferencias" (MEN, 2013, p. 14). Cómo llegar a ese modelo de sociedad es todo un reto que implica diseñar estrategias pedagógicas que sean verdaderamente útiles en la formación de los estudiantes como sujetos socio-políticos, desde las Competencias Ciudadanas; para que sean capaces de respetar la pluralidad, las diferencias y avanzar hacia una sociedad cada vez más civilizada. Dichas estrategias estarán sustentadas en fundamentos teóricos desde la Filosofía política y la Ciencias Sociales, aportando razones para creer que un mundo diferente puede ser posible si se propone trabajar por él, esa sociedad donde reine la convivencia.

Se entiende por convivencia como la acción de convivir (vivir en compañía de otro u otros). En su acepción más amplia, se trata de un concepto vinculado a la coexistencia pacífica y armoniosa de grupos humanos en un mismo espacio (Balanta, Muñoz y Torres, 2015, p.39). Sin embargo, los autores señalan que, a su vez, no es sencilla la forma en se llega a esa coexistencia, así. El ser humano es un ser social. Ninguna persona vive absolutamente aislada del resto, ya que la interacción con otros individuos es imprescindible para el bienestar y la salud. Sin embargo, la convivencia no siempre resulta fácil, dado que pueden interferir negativamente ciertas diferencias sociales, culturales o económicas, entre otras muchas posibilidades (Balanta, Muñoz y Torres, 2015, p. 40).

Entonces, de acuerdo a este planteamiento el respeto y la solidaridad son dos valores imprescindibles para que la convivencia armoniosa sea posible. (Balanta, Muñoz y Torres, 2015), valores que la mayoría de las veces son difíciles de hallar en los terrenos no se siembra y crece la política. De acuerdo a esto, la Guía $\mathrm{N}^{\circ} 49$, al definir la convivencia escolar sostiene que: "se puede entender como la acción de vivir en compañía de otras personas en el contexto escolar y de manera pacífica y armónica" (MEN, 2013, p. 25).

Reafirmando este ideal, Antanas Mockus, toda una autoridad en materia de Competencias Ciudadanas por la forma en que se ilustra y llevó a la práctica sus conocimientos durante su ejercicio como burgomaestre de la ciudad de Bogotá, sostiene: "La convivencia escolar resume el ideal de la vida en común entre las personas que forman parte de la comunidad educativa, partiendo del deseo de vivir juntos de manera viable y deseable a pesar de la diversidad de orígenes". (MEN, 2013, p.25).

En cualquier contexto formativo como expresan Araque y Suarez (2017), la acción educativa fundada en la responsabilidad ética puede promover una sociedad del saber, que oriente al conocimiento con estrategias de inclusión social.

En estos casos es primordial "utilizar estos conflictos para que todas las personas que conforman la comunidad educativa haga uso del diálogo como opción para transformar las relaciones; el pensamiento crítico como una opción para entender lo que ocurre". (MEN, 2013, p.25); teniendo en cuenta lo planteado, una mejor convivencia escolar debe propiciar un mejor desarrollo de los procesos de democráticos 
que tengan como principios rectores el uso de la razón y la definición de consensos.

La forma eficaz del entendimiento entre seres humanos en esa sociedad compartida es el diálogo; basado en ello el diálogo entre los actores es fundamental para construir un equilibrio entre el cuidado, la justicia y la veracidad, evitando así las soluciones emergentes e inmediatistas que clausuran los contrastes. (Kröyer, Muñoz y Gajardo, 2013, p. 382). En esa replica de sociedad que son los miembros que integran un aula escolar, a saber, docentes y estudiantes, el uso del diálogo como uno de los máximos triunfo de la razón y la acción humana, es la vía más segura para hacer uso del poder de la palabra.

Por lo anterior, se dirige la mirada ahora hacia autoridades en la materia, quienes, desde la Filosofía política, más aún, desde la teoría critica de la sociedad, aportan a la investigación una lumbrera que guía ese sendero que se desea transitar para hallar las claves del diseño de estrategias pedagógicas que sean capaces de formar a los estudiantes como sujetos socio-políticos. Se sabe que es desde la competencia ciudadana de donde debe emerger ese sujeto político, dueño de unas habilidades con las que pueda desarrollar de manera armónica su vida en sociedad, más pertinente aún, su vida política; basada en principios democráticos, respetuoso de las diferencias, siendo capaz de convivir con ellas. Pero también se desea formar a ese mismo sujeto con una habilidad especial: la de intervenir para lograr la transformación de la sociedad en la que desarrolla su vida, para hacerla cada vez mejor, para sí, y para los que viene después de él.

Por su parte la Investigación como Estrategia Pedagógica, tiene como finalidad instaurar bases científicas a los estudiantes en el aula de clases, esta parte de una dinámica metodológica, que busca dar respuestas a los interrogantes de los niños y niñas de la Institución y que asigna a los docentes un nuevo rol en el aula, para que en el momento indicado, puedan ser ellos mismos quienes busquen comprender el mundo que los rodea e interpretar y describir sus problemas en un lenguaje sustentado en la ciencia. En la implementación de la IEP, se parte de la pregunta del sentido común de los niños, niñas y jóvenes para reelaborarla en la perspectiva de la educación popular, que reconoce la existencia de saberes comunes y elaborados así en la negociación con los conocimientos disciplinares, y de las maneras como se correlacionan en la negociación cultural (Mariño, 2010),

La educación a lo largo del tiempo ha presentado cambios significativos, que invitan al docente a diseñar e implementar nuevas estrategias pedagógicas para dirigir el proceso de enseñanza con sus estudiantes, una estrategia pedagógica es un conjunto de actividades que se realizan con un orden lógico y coherente en función del cumplimiento de objetivos del plan académico. Es decir, es una planificación que contiene métodos o acciones que permiten al estudiante alcanzar los logros propuestos, mejorar su aprendizaje y facilitar su crecimiento personal (Picardo, Balmore, y Escobar, 2004).

En ese sentido, pensar la investigación desde las corrientes educativas criticas significa una lucha teórico-práctica por la manera como sus presupuestos sobre el conocimiento, la ciencia, su epistemología, la cultura, lo humano y los grupos sociales enmarcan una acción que durante cuatrocientos años ha sido señalada como objetiva, y que no solo ha construido una forma de ella, sino que también ha ayudado a generar formas de poder que en la sociedad han servido para el control y la gestación de desigualdades, y en estos tiempos, nuevas formas de acumulación y dominación (Ortega, 2009).

Una de las finalidades de la educación es capacitar a los estudiantes para comprender, crear y participar en la construcción de 
su conocimiento, para lo anterior el docente debe cumplir un rol de guía o formador, que a través de estrategias pedagógicas motive al estudiante a alcanzar los logros propuestos, incluir en dichas estrategias las TIC, servirá como una herramienta tecnológica que muestra una forma diferente de organizar, representar y codificar la realidad, además estas son instrumentos que permiten un grado de aplicación de los conocimientos adquiridos. Las tecnologías del aprendizaje representan una de las fuerzas renovadoras en los sistemas de aprendizaje y constituyen un elemento clave para el desarrollo de la educación y la formación (Oleagordia Aguirre, s.f).

Las TIC conforman el proceso del manejo de la información incluyendo medios y herramientas de manipulación, las TIC se convirtieron en un elemento esencial en los procesos educativos, en la actualidad el internet se implanto como la principal fuente para acceder a la información, donde las herramientas tecnológicas como computadores, tablets y celulares, son una herramienta necesaria para almacenarla y procesarla (Camacho., Flórez., Gaibao., Aguirre., Pasive., y Murcia, G. 2012).

Así mismo, las TIC son tecnologías para almacenar, recuperar, procesar y comunicar la información. Existe una variedad de recursos electrónicos que se encuadran dentro del concepto de TIC como; televisión, teléfonos, videos, ordenadores, entre otros. Sin embargo, los medios más representativos de la sociedad actual son los ordenadores que nos permiten utilizar diferentes aplicaciones informáticas (presentaciones, aplicaciones multimedia, programas ofimáticos y redes de comunicación (Belloch, 2012).

No obstante, el uso de las TIC no implica la implementación de una estrategia de enseñanza/aprendizaje. En algunos casos se producen procesos educativos que integran las TIC siguiendo una metodología tradicional en la que se enfatiza el proceso de enseñanza, donde el estudiante recibe la información del docente. No obstante, para guiar el proceso educativo, se puede fomentar la interacción, el aprendizaje colaborativo y el aprendizaje por descubrimiento utilizando las TIC basados en los diferentes recursos y servicios que ofrece Internet. (Belloch, 2012).

En ese sentido la IEP apoyada en las TIC es una propuesta educativa que apunta a mejorar la calidad de la educación, presentando una formación en ciencia, tecnología e innovación. Que permiten el desarrollo de competencias y la creación de nuevo saber y conocimiento el cual va a generar estrategias diversas para afrontar los conflictos escolares, donde a partir de la transversalización de las áreas de estudio las instituciones educativas a través de una metodología didáctica y práctica, favorecen e impactan de manera positiva el proceso enseñanza - aprendizaje, mediante el fortalecimiento de la convivencia escolar.

\section{Metodología}

El trabajo de investigación es de enfoque cualitativo, de tipo Investigación Acción Participación (IAP). La investigación cualitativa busca conocer e interpretar la realidad de los participantes a través de sus propias experiencias, entregando una información subjetiva del fenómeno de estudio. (Hernández, Fernández, y Baptista, 2010). En la IAP el conocimiento de la realidad no se descubre ni se posee es el resultado de la trasformación subjetiva y objetiva de el mismo proceso de investigación, en consideración a esta la validez del conocimiento se da por la capacidad que posee para orientar la trasformación de una comunidad o grupo mejorando la calidad de vida de sus integrantes. La IAP es un proceso educativo de autoformación en el cual las personas que pertenecen al grupo sobre quienes esta la realidad estudiada, tienen una participación directa en el pro- 
ceso de investigación y en la producción de conocimiento de su realidad.

En cuanto a los instrumentos de recolección se utilizó el diario de campo, este es un instrumento que ayuda al investigador a inmiscuirse de forma total en el ambiente con el cual se va a encontrar, es un proceso de hechos, recolección de datos y análisis. El investigador entiende a los participantes y no se dedica únicamente a registrar "hechos". El diario de campo es parte de un registro cronológico que se desarrolla mediante la sesión compartida con los infantes, es donde se describe detalladamente los aspectos positivos como negativos colocando significado a cada uno de ellos como un recurso de mejoras en todas las áreas con el grupo a trabajar considerando los recursos manejados y utilizados. Un cuaderno donde se va a descubrir la manera de expresar y escribir frente a la relación que se tiene con los infantes, es un momento de no cohibir lo vivido y las experiencias obtenidas. Se tomó una muestra representativa de sesenta (60) estudiantes de sexto grado con edades comprendidas entre diez (10) y trece (13) años de la institución educativa Antonio Bruges Carmona del municipio de Santa Ana - Magdalena.

\section{Resultados}

De la información recolectada se hizo una reconstrucción cómo fueron esos años en que niños y adolescentes vieron en estos espacios democráticos una oportunidad para imitar lo que los adultos hacen en tiempo electoral. A medida que pasó el tiempo los vicios políticos se apoderaron de los ambientes escolares y se hacía sentir en los procesos eleccionarios. En lo correspondiente a la actuación de los estudiantes, la cosa comenzó a distanciarse de los propósitos institucionales.

En complemento con el primer propósito de la IAP se elaboró una construcción bibliográfica que conllevó a la construcción del marco teórico. De acuerdo a los autores estudiados se obtuvo fundamentos teóricos que soportan la investigación bajo esa comprensión de que el ser humano es el resultado de aquello que han hecho de él. Bajo esta luz se pudo notar que, tal como contemplan los teóricos y, en algunos casos, los complementos experimentales, los estudiantes actúan conforme los han educado en los diferentes contextos en donde tiene participación. De alguna manera, se apropian de prácticas que les son dadas, muchas veces sin que las pida; todo funciona como una especie de sistema que los absorbe, los moldea y los pone actuar de la manera en que todo funciona.

Se pasó de lo teórico a lo práctico estimulando una formación que apunta a formar un sujeto político-social que pone en práctica unas competencias ciudadanas con las que logra cambiar la forma en que procede la sociedad actual. cuidando a las semillas de ciudadanos, educándolos, como seres humanos críticos, creando oportunidades de transformar desde lo interno, a nivel institucional a ese contexto externo; abriendo los espacios necesarios para que el cambio sea posible.

El espacio generado por la IED Antonio Brugés Carmona, ha sido propicia para llevar a la práctica lo adelantado hasta el momento; se pudo constatar que la sociedad sería mucho mejor si hiciéramos uso de la razón y la palabra para desarrollar la práctica política. Ese pequeño campo de experimentación que es el plantel educativo, le permitió al grupo investigador hacer evidenciable de que la transformación es posible si creamos los espacios y las estrategias apropiadas para formar $y$ convencer a los estudiantes Brugesistas de que las cosas en materia política pueden ser diferentes.

Describir como era el proceso de elección del personero escolar en la institución antes de esta investigación, era describir 
a pequeña escala lo que acontece en el plano municipal en un proceso de elecciones de alcalde y concejales. Hoy, gracias al proyecto de investigación, la situación acontece de manera distinta, al aplicar estrategias pedagógicas orientadas a la generación de espacios, prácticas políticas y democráticas, marcadas por la honestidad y uso de la razón.

En este orden de ideas, los estudiantes aspirantes participaron de una estrategia pedagógica que incluyó la realización de conversatorios en cada aula escolar donde deliberaron sobres diversos temas relacionados con el cargo al que aspiran, las necesidades del estudiante y la institución. En ningún momento se muestran como opositores, transmiten fraternidad al estudiantado y en los conversatorios hacen buen uso de la razón y la palabra, dejando libre la decisión de elegir a quien consideren tiene el mejor perfil para el cargo. Las campañas distan mucho de lo que en otrora se efectuaba; la elección se realiza en cada aula escolar con un voto inteligente, que enseña a decidir, no a marcar. Posterior a los resultados no hay celebración, todos hacen su esfuerzo en mostrar que ha ganado la institución por desarrollar un proceso de esa calidad. Se contrarresta el matoneo a quienes no resultan ganadores.

Estas acciones se ven complementadas con la participación del estudiantado en eventos académicos e intelectuales, donde tiene el contacto y la interacción con líderes políticos que son o han sido alcaldes, concejales o, en su defecto han actuado como candidatos a corporaciones públicas. De esta manera, se lleva la política a los terrenos de la academia, y se respira una democracia basada en la deliberación, los argumentos afilados y el consenso.

En lo que respecta a ir saliendo de la institución para lograr contar con la reconstrucción de la forma actual en que se ha desarrollado la política en el municipio, se participó en la realización de videos documentales adelantados por estudiantes, en donde se recogieron testimonios compartidos de líderes políticos de tradición en el municipio que relataban cómo se ha desarrollado los eventos eleccionario a través de la historia santanera, en qué tipo de vicos electorales han participado ellos, confrontándolos con las ideas de los miembros del grupo de investigación y líderes políticos que comienzan a creer en el cambio.

Se sentaron las bases para construir una verdadera democracia, a partir del cambio en la Cultura política, sirviendo como referentes para futuras investigaciones que permitan incidir en esa realidad socio-política más allá de las paredes institucionales; por ejemplo, con aquellas en las que se puede formar y evidenciar un estudiante que actúa como sujeto sociopolítico, en actividades como: la emisión del programa radial "FiloGenios hacia la Politeia”, la edición de la Revista ABC Intelecto; complementadas con la socialización del proyecto de investigación en espacios valiosos como: el Consejo de Política Social del Municipio de Santa Ana, Magdalena, y el Concejo Municipal de Santa Ana; llevando esta propuesta de cambio más allá de las fronteras municipales, llevando este sueño al Colegio "Octavio Daza Daza”, de la ciudad de Valledupar, Cesar.

Las Tic han sido fundamentales en la divulgación del proyecto de investigación, sobre todo en la grabación y edición de videos y el uso de las redes sociales; hoy FiloGenios hacia la Politeia, posee cuentas en redes sociales como: Facebook y YouTube, facilitando así la divulgación de los ideales del Grupo de investigación. Del mismo modo, la creación de la historieta super politeio, comic que pretende hacer comprensible el proyecto de investigación a niños y jóvenes, y convencerlos de que: "Un cambio en la Cultura política, Sí es posible". 


\section{Conclusiones}

Acciones como las descritas anteriormente han permitido diseñar la ruta que se perfila al diseño de las estrategias pedagógicas orientadas a la formación de los estudiantes como sujetos socio-políticos, desde las competencias ciudadanas; sustentados en presupuestos filosófico-políticos como: los principios de la democracia deliberativa, que promueve el entendimiento de los ciudadanos a través del diálogo racional, respetando las diferentes perspectivas, pero procurando llegar a puntos de encuentro en beneficio del bien común; además, el concepto del uso público de la razón y la intervención de los intelectuales públicos en los debates de carácter relevante en la sociedad, agregando también, los acercamientos a la teoría de la comunicación para el cambio social, que se sustenta en los conceptos claves de ciudadanía y esfera pública política.

De ese modo, se puede concluir que, de acuerdo al estudio de experiencias similares en ciudades de Colombia, es posible un cambio de mentalidad en diferentes aspectos, teniendo como eje generador de cambio a la educación; logrando crear cultura ciudadana para tener una mejor relación entre gobernantes y ciudadanía, ahorrar agua, mejorar en la convivencia, respetar las normas de tránsito, comprender el concepto de los bienes públicos, entre otros. Actividades como estas han logrado que los estudiantes y también docentes investigadores crean en que el cambio puede ser posible, teniendo la claridad que el aspecto a trabajar por parte de los "FiloGenios hacia la Politeia", es más complejo por tratarse de política.

Una acción enriquecedora como esta, ha generado el deseo de convertirse en protagonistas del cambio, siendo conscientes de lo difícil que resultará lógralo, pero en la medida en que se avanza también en las lecturas de análisis de teoría política, va creciendo el conocimiento y las ganas de experimentar lo que pude ser una forma de garantizar una sociedad ideal para las generaciones venideras.

\section{Referencias}

Araque, F. y Suárez, O. (2017). Reflexiones teóricas y legales del adulto mayor y la discapacidad en Colombia. JURÍDICAS CUC, 13(1). 97-120. http://dx. doi. org/10.17981/juridcuc.13.1.2017.5

Balanta, A., Muñoz, F. y Torres, H. (2015). Actividades lúdicas encaminadas al fortalecimiento de la convivencia escolar en los alumnos del grado quinto de la Institución Educativa Técnico Comercial Villa de la Sur sede Susana Vinasco de Quintana. [Trabajo de Grado]. Especialización pedagogía de la Lúdica. Fundación Universitaria los Libertadores, Bogotá, D.C.

Belloch. (2012). Las Tecnologías de la Información y Comunicación en el aprendizaje. Universidad de Valencia. Recuperado de https://www.uv.es/bellochc/pedagogia/EVA1.pdf

Camacho, T., Flórez M., Gaibao, D., Aguirre M., Pasive Y. y Murcia, G. (2012) Estrategias pedagógicas en el ámbito educativo. Bogotá, D.C.

Colombo, G. (2011) Violencia Escolar y Convivencia Escolar: Descubriendo estrategias en la vida cotidiana escolar. Revista Argentina de Sociología, 8(9). 15-16,

Gallardo, V. (2009). Educación ciudadana y convivencia democrática. Revista interuniversitaria de pedagogía social -SIPS, 16. 119-133.

Guzmán, E. y Preciado, A. (2012). La convivencia escolar: una mirada desde la diversidad cultural. [Tesis de Maestría]. Universidad de Manizales. Caldas, Manizales.

Hernández, G., Alvarado, L. y Chumaceiro H. (2013). Estado, gestión pública y participación ciudadana desde la modernidad y posmodernidad. Revista Gestión y Gerencia, 7(1). 66-80 . 
Hernández, R., Fernández, C., y Baptista, P. (2006). Metodología de la investigación. $4^{\mathrm{a}}$ Edición. México, D.F.: McGrawHill.

Mariño, G. (2010). El diálogo en la educación de jóvenes y adultos. Dos propuestas pedagógicas para implementarlo. En, $E l$ taller dialógico / la recuperación de experiencias laborales. Bogotá, D.C.: OEI.

Mockus, A. (2004). Formación en cultura ciudadana: la experiencia bogotana. En, Foro Educativo Nacional Competencias ciudadanas. (33-52). Bogotá, D.C.: Ministerio de Educación Nacional

Muñoz, M. y Gajardo, J. (2013). Principios orientadores de la convivencia en el aula: una estrategia de reflexión colectiva. Educ. Pesqui., São Paulo, 39(2). 367-385.

Navarro, L. (2011). Entre esferas públicas y ciudadanía. Barranquilla: Editorial Universidad del Norte.

Oleagordia, I. (s.f.). Estrategias educativas para el uso de las nuevas tecnologías de la información y la comunicación. Revista Iberoamericana de la educación, 9.

Ochoa, R. (2015). Comportamiento Electoral de los Colombianos durante las elecciones presidenciales del año 2010. Jurídicas CUC, 11(1), 209-220. http:// dx.doi.org/10.17981/juridcuc.11.1.2015.9

Ortega, P., Peñuela, D. y López, D. (2009). Sujetos y prácticas de la pedagogía crítica. Bogotá, D:C.: Ediciones El Búho.

Picardo, O., Balmore, R., \& Escobar, J. (2004). Diccionario enciclopédico de ciencias de la educación. San Salvador: El Salvador.

República de Colombia. Congreso de la República. (febrero 8 de 1994). Por la cual se expide la ley general de educación. [Ley 115]. Diario Oficial No. 41.214. Recuperado de https://www.mineducacion. gov.co/1621/articles-85906_archivo_pdf. pdf
República de Colombia. Presidencia de la República. (agosto 5 de 1994). Por el cual se reglamenta parcialmente la Ley 115 de 1994, en los aspectos pedagógicos y organizativos generales. [Decreto 1860]. Diario Oficial No. 41.473.

República de Colombia. Congreso de la República. (septiembre 11 de 2013). Por la cual se crea el sistema nacional de convivencia escolar y formación para el ejercicio de los derechos humanos, la educación para la sexualidad y la prevención y mitigación de la violencia escolar. [Ley 1620]. Diario Oficial No. 48.733 Recuperado de http://wsp.presidencia.gov.co/Normativa/Leyes/Documents/2013/LEY\% $201620 \% 20$ DEL\%2015\%20DE\%20MARZO\%20 DE\%202013.pdf

República de Colombia. Ministerio de Educación Nacional. (2005). Estándares Básicos de Competencias Ciudadanas. Bogotá, D:C.: Mineducación. Recuperado de https://www.mineducacion.gov.co/cvn/1665/articles-75768_ archivo_pdf.pdf

República de Colombia. Ministerio de Educación Nacional. (2013). Guía 49. Guías pedagógicas para la convivencia escolar. Ley 1620 de 2013 - Decreto 1965 de 2013. Bogotá, D:C.: Mineducación. Recuperado de http://redes.colombiaaprende.edu.co/ntg/men/pdf/Guia\%20 No.\%2049.pdf

Rojas, J. (2016). Intelectual público; el rol del filósofo contemporáneo, desde la perspectiva de Jürgen Habermas. Bogotá, D:C.: Autores editores. 